\title{
An Implementation of Combined Local-Global Optical Flow
}

\author{
Jorge Jara-Wilde ${ }^{1}$, Mauricio Cerda ${ }^{2}$, José Delpiano ${ }^{3}$, Steffen Härtel ${ }^{2}$ \\ ${ }^{1}$ DCC, SCIAN-Lab, BNI, University of Chile, Chile (jjara@dcc.uchile.cl) \\ ${ }^{2}$ SCIAN-Lab, ICBM, BNI, University of Chile, Chile (mauriciocerda, shartel@med.uchile.cl) \\ ${ }^{3}$ University of the Andes, Chile (jdelpian@uandes.cl) \\ Communicated by Enric Meinhardt-Llopis Demo edited by Enric Meinhardt-Llopis
}

\begin{abstract}
Optical Flow (OF) approaches for motion estimation calculate vector fields for the apparent velocities of objects in image sequences. In 1981 Horn and Schunck (HS) introduced two basic assumptions: "brightness value constancy" and "smooth variation" to estimate a smooth OF field over the entire image -global approach-. In parallel, Lucas and Kanade (LK) assumed constant motion patterns for image patches, estimating piecewise-homogeneous OF fields -local approach-. Several variations of these approaches exist today. Here we present the combined local-global (CLG) approach by Bruhn et al. which encompasses properties of HS-OF and LK$\mathrm{OF}$, aiming to improve the OF accuracy for small-scale variations, while delivering the HS-OF dense and smooth fields. A multiscale implementation is provided for 2D images, together with two numerical solvers: Successive Over-Relaxation and the faster Pointwise-Coupled GaussSeidel by Bruhn et al.. The algorithm works on gray-scale (single channel) images, with color images being converted prior to the OF computation.
\end{abstract}

\section{Source Code}

The source code (ANSI C), its documentation, and the online demo are accessible at the IPOL web page of this article ${ }^{1}$.

\section{Supplementary Material}

Sample images and the CLG-OF demo are available here ${ }^{2}$.

Keywords: motion estimation; optical flow

\footnotetext{
${ }^{1}$ https://doi.org/10.5201/ipol.2015.44

${ }^{2}$ https://doi.org/10.5201/ipol.2015.44
} 


\section{Introduction}

In this article we describe the combined local-global optical flow approach introduced by Bruhn et al. [3]. A review of the OF approaches from Horn \& Schunck and Lucas \& Kanade is also provided, as their constraints have been encompassed by the CLG-OF formulation. We also present the description for the CLG-OF numerical solution, including (i) a multiscale strategy to increase the detectable motion range of OF methods, and (ii) two numerical solvers: Successive Over-Relaxation and the faster Pointwise-Coupled Gauss-Seidel.

The article is organized as follows. In the remainder of this section, an overview of the HS-, LK- and CLG-OF methods is presented, together with a standard multiscale approach. Section 2 introduces the CLG-OF numerical solution, considering the discrete equations and the two solvers implemented. Section 3 presents the implemented algorithm pseudocode, function descriptions, and complexity analysis. Section 4 provides example results of the presented implementation, together with error evaluations in well known OF database sequences.

It must be noted that, even when the CLG-OF parameters are set to equal the HS-OF model (by setting $\rho=0$ ), the results from this implementation often yield worse error metrics compared to other IPOL HS-OF versions. Other than the number of scales and parameter values, we have found that the main reason accounting for this difference is the stage at which the linearization (image warping) is performed. Other HS-OF IPOL implementations perform late linearization, while ours performs early, as in the original article by Bruhn et al. (see Subsection 1.4). It is known that late linearization improves OF results for large displacements; however, it was not originally proposed for the HS or CLG methods. We opted for keeping the implementation close to the original approach.

\subsection{Horn-Schunck Global Approach}

Let $I=I(x, y, t)$ the input image sequence (two or more images), and $\underline{V}=[u(x, y, t), v(x, y, t), 1]^{T}$ the optical flow vector field. The HS model assumes that the image total brightness level is constant across time, and that the estimated flow vectors vary smoothly across the image space. The brightness constancy assumption is expressed by making the time derivative $I_{t}=d I / d t=0$. The HS-OF [8] is then defined from a first-order Taylor expansion (linearization):

$$
I(x+u, y+v, t+\Delta t)-I(x, y, t)=0 \Longrightarrow I_{x} u+I_{y} v+I_{t}=0 .
$$

Integrating over the image domain $\Omega$, an "energy functional" is defined as

$$
E_{H S}(\underline{V})=\int_{\Omega}\left(I_{x} u+I_{y} v+I_{t}\right)^{2}+\alpha\left(|\nabla u|^{2}+|\nabla v|^{2}\right) d x d y
$$

with $I_{x}, I_{y}$ the spatial derivatives, and $|\nabla u|,|\nabla v|$ the norms of the gradient vectors for $u$ and $v, \nabla u$ and $\nabla v$, respectively. $\alpha\left(|\nabla u|^{2}+|\nabla v|^{2}\right)$ acts as a smoothing constraint for the OF field, since large variations in $\underline{V}$ account for an increase in the magnitude of $\nabla u$ and $\nabla v$. The weight coefficient $\alpha>0$ is henceforth called global regularization coefficient. Higher values of $\alpha$ yield more homogeneous fields, while lower values allow more dissimilar displacement vectors. It must be noted that this coefficient is denoted by $\alpha^{2}$ in the HS-OF article [8], instead of $\alpha$ in the CLG-OF article [3].

\subsection{Lucas-Kanade Local Approach}

The LK approach [10] assumes that the flow is constant within a neighborhood of size $\rho$, computing the OF field for all the pixels in that neighborhood, by the least squares criterion:

$$
E_{L K}(u, v)=G_{\rho} *\left(I_{x} u+I_{y} v+I_{t}\right)^{2} .
$$


where $G_{\rho} *$ denotes convolution with a Gaussian kernel of size $\rho$. A minimum $(u, v)$ for $E_{L K}$ satisfying $\partial_{u} E_{L K}=0$ and $\partial_{v} E_{L K}=0$ yields the following system of equations:

$$
\left[\begin{array}{cc}
G_{\rho} *\left(I_{x}^{2}\right) & G_{\rho} *\left(I_{x} I_{y}\right) \\
G_{\rho} *\left(I_{x} I_{y}\right) & G_{\rho} *\left(I_{y}^{2}\right)
\end{array}\right]\left[\begin{array}{l}
u \\
v
\end{array}\right]=\left[\begin{array}{l}
-G_{\rho} *\left(I_{x} I_{t}\right) \\
-G_{\rho} *\left(I_{y} I_{t}\right)
\end{array}\right]
$$

If the image gradients are not zero then the system matrix is invertible, yielding a unique solution.

\subsection{Bruhn et al. Combined Local-Global Approach}

Bruhn et al. [3] defined the CLG-OF by an integral functional that encompasses both the HS and the LK approaches. This combined approach aims to produce dense flow fields (characteristic of the global approaches) which are robust against noise, by employing smoothing terms based on both the global and local approaches.

Let $\nabla_{3} I=\left[I_{x}, I_{y}, I_{t}\right]^{T}$, then the HS-OF energy functional can be written as:

$$
E_{H S}(\underline{V})=\int_{\Omega}\left(\underline{V}^{T}\left(\nabla_{3} I \nabla_{3} I^{T}\right) \underline{V}+\alpha\left(|\nabla u|^{2}+|\nabla v|^{2}\right)\right) d x d y .
$$

The CLG-OF energy functional introduces a smoothing term in $\nabla_{3} I$. It is defined as

$$
E_{C L G}(\underline{V})=\int_{\Omega}\left(\underline{V}^{T} J_{\rho}\left(\nabla_{3} I\right) \underline{V}+\alpha\left(|\nabla u|^{2}+|\nabla v|^{2}\right)\right) d x d y
$$

with $J_{\rho}\left(\nabla_{3} I\right)=G_{\rho} *\left(\nabla_{3} I \nabla_{3} I^{T}\right) . J_{\rho}$ acts as a local spatio-temporal derivative smoothing term, defined as a convolution $*$ between a bi-dimensional Gaussian kernel $G_{\rho}$, with standard deviation $\rho$, with the matrix $\left(\nabla_{3} I \nabla_{3} I^{T}\right)$. If $\rho=0$ no local smoothing occurs, making the CLG-OF functional equal to the HS-OF. If $\alpha=0$ the functional becomes equivalent to the LK-OF.

\subsection{Multiscale Optical Flow}

In order to fulfill the OF assumption, HS and CLG approaches impose a linearization of the brightness constancy constraint, thus the vector field $\underline{V}$ is required to be small. This can be a problem for image sequences with large object displacements that can appear as discontinuities and lead to erroneous solutions. To address this issue, multiscale (MS) strategies have been proposed [4, 11], using a coarse-to-fine approach to successively compute more accurate OF vector fields.

The main idea of the MS strategy is to compute an image pyramid with decreasing size for each image in the set where $\mathrm{OF}$ is going to be estimated (typically two frames, $I_{1}$ and $I_{2}$, at times $t_{1}$ and $t_{2}$ ). Starting from the smallest (coarsest) image of the pyramid, the OF is computed, and for the next (finer) scales, the coarse estimation is used to "warp" the second frame at $t_{2}$, denoted as $I(x+\delta \underline{V})$ and compute the corresponding OF field. Thus, at each level of the pyramid the OF increment is computed $(\delta \underline{V})$, and the final OF estimation is the interpolated sum of the estimations from all the pyramid levels. The MS CLG-OF can be written as:

$$
E_{C L G}\left(\underline{\delta V^{m}}\right)=\int_{\Omega}\left(\underline{\delta V^{T}} J_{\rho}\left(\nabla_{3} I(x+\delta \underline{V})\right) \underline{\delta V}+\alpha\left(|\nabla \delta u|^{2}+|\nabla \delta v|^{2}\right)\right) d x d y,
$$

where $\delta \underline{V}=(\delta u, \delta v)$. In general, the MS strategy can be applied to any OF algorithm, by considering two additional parameters: the total number of scales and the scaling factor.

It is worth note that other IPOL OF methods available [11, 12] perform late linearization which yields better accuracy for larger displacements $\underline{d}=\left[d_{x}, d_{y}\right]$, by making $I_{1}(x, y)-I_{2}\left(x+d_{x}, y+d_{y}\right)=0$ instead of the right side in Equation (1). In MS CLG-OF, the warping of the second image is carried out only once per scale. As can be observed in those IPOL OF publications, an outer loop is used to solve the system several times, updating the value of $I(x+\delta \underline{V})$ in each iteration, and so improving the OF accuracy. 


\section{Numerical Solution Schemes}

The minimum of the CLG-OF energy functional must satisfy the Euler-Lagrange equation, in the form of a system of partial differential equations

$$
\begin{aligned}
& \alpha \Delta u-\left(J_{11} u+J_{12} v+J_{13}\right)=0, \\
& \alpha \Delta v-\left(J_{21} u+J_{22} v+J_{23}\right)=0,
\end{aligned}
$$

with $J_{i k}$ denoting the elements of the matrix $J_{\rho}$. Neumann boundary conditions are assumed

$$
\partial_{n} u=0, \partial_{n} v=0 .
$$

Although the CLG-OF can become equivalent to the HS-OF depending on the parameter values, different discretization schemes were used in the original articles, leading to slightly different results when both methods are applied to the same set of images. This also occurs for other OF approaches and numerical schemes, as shown by Delpiano et al. [5] and Hubený et al. [9] for fluorescent moving objects in microscopy images. See Sun et al [13] for a general overview.

For computing $J_{\rho}$, the following discrete derivatives are used:

$$
\begin{array}{r}
\left.I_{x} \approx I * \frac{1}{2 h}\left[\begin{array}{lll}
-1 & 0 & 1
\end{array}\right]_{x}\right|_{x_{i}, y_{j}, t_{k},} \\
\left.I_{y} \approx I * \frac{1}{2 h}\left[\begin{array}{lll}
-1 & 0 & 1
\end{array}\right]_{y}\right|_{x_{i}, y_{j}, t_{k}}, \\
I_{t} \approx I\left(x_{i}, y_{j}, t_{k+1}\right)-I\left(x_{i}, y_{j}, t_{k}\right),
\end{array}
$$

with $h$ the step size of the discrete domain ( $h=1$ for one pixel, for instance), and $\left.A_{l}\right|_{x_{i}, y_{j}, t_{k}}$ the mask vector $A$ applied along the $l$ axis at image position $\left(x_{i}, y_{j}, t_{k}\right)$ (i.e. the central element of the mask corresponds to the pixel $\left.\left(x_{i}, y_{j}, t_{k}\right)\right)$. For time derivatives, a warped version of $I\left(x_{i}, y_{j}, t_{k+1}\right)$ is used. Then, $J_{\rho}$ is computed as follows:

1. $J_{0}=\nabla_{3} I \nabla_{3} I^{T}$

2. if $\rho=0$ then $J_{\rho}=J_{0}$; otherwise

3. a square Gaussian matrix $G_{\rho}$ with standard deviation $\rho$ is computed; and

4. $J_{\rho}=J_{0} * G_{\rho}$.

The Laplacian is computed by a convolution between the OF components and a kernel matrix $M$,

$$
\begin{aligned}
M & =\left[\begin{array}{ccc}
0 & 1 & 0 \\
1 & -4 & 1 \\
0 & 1 & 0
\end{array}\right], \\
\Delta u_{x_{i}, y_{j}, t_{k}} & \left.\approx u * M\right|_{x_{i}, y_{j}, t_{k}}, \\
\Delta v_{x_{i}, y_{j}, t_{k}} & \left.\approx v * M\right|_{x_{i}, y_{j}, t_{k}} .
\end{aligned}
$$

The coupled system in equations (8)-(9) can be written as:

$$
\begin{aligned}
& \alpha u_{x_{i}, y_{j}, t_{k}} * M-J_{11} u_{x_{i}, y_{j}, t_{k}}=-\left(J_{13}+J_{12} v_{x_{i}, y_{j}, t_{k}}\right), \\
& \alpha v_{x_{i}, y_{j}, t_{k}} * M-J_{22} v_{x_{i}, y_{j}, t_{k}}=-\left(J_{23}+J_{21} u_{x_{i}, y_{j}, t_{k}}\right) .
\end{aligned}
$$

This coupled system can be solved by different numerical schemes. For this work we implemented two iterative schemes to solve equations (16)-(17), also used by Bruhn et al. [2, 4]: Successive Over-Relaxation (SOR), which solves sequentially $u_{x_{i}, y_{j}, t_{k}}$ and then $v_{x_{i}, y_{j}, t_{k}}$, and Pointwise-Coupled Gauss-Seidel (PCGS), which simultaneously solves $\left(u_{x_{i}, y_{j}, t_{k}}, v_{x_{i}, y_{j}, t_{k}}\right)$ at each pixel. 


\subsection{Successive Over-Relaxation Scheme}

Equations (16)-(17) can be solved as a linear system in the form $A x=b$ ( $x$ being either $u$ or $v$ ), where the matrix $A$ is decomposed in the form $A=D-L-U$, with $D$ a diagonal matrix and $L, U$ lower and upper triangular matrices, respectively. From a given initial value $x^{0}$, the equation is solved iteratively until a convergence criterion is reached. The $k$-th SOR iteration for $x$ is given by $x^{k+1}=(D-L)^{-1}\left(U x^{k}+b\right)$. In this case, two equations must be solved for the OF field components, $(u, v)$ as described next. For these equations the subscript notation is changed from two indexes (e.g. $u_{i j}$ ) to one index $\left(u_{i}\right)$, in order to be consistent with the original CLG-OF article [4].

Let $U$ and $V$ be the matrices storing the $x$ and $y$ components of the OF field at given position $i$ and iteration $k$, the values of the OF field $\left(u_{i}, v_{i}\right)$ for the next iteration are computed as

$$
\begin{gathered}
u_{i}^{k+1}=(1-w) u_{i}^{k+1}+w \frac{\sum_{l=1}^{2} \frac{\alpha}{h_{l}^{2}}\left(\sum_{j \in N_{l}^{-}(i)} u_{j}^{k+1}+\sum_{j \in N_{l}^{+}(i)} u_{j}^{k}\right)-\left(J_{12 i} v_{i}^{k}+J_{13 i}\right)}{\sum_{l=1}^{2} \frac{\alpha}{h_{l}^{2}}\left|N_{l}(i)\right|+J_{11 i}}, \\
v_{i}^{k+1}=(1-w) v_{i}^{k+1}+w \frac{\sum_{l=1}^{2} \frac{\alpha}{h_{l}^{2}}\left(\sum_{j \in N_{l}^{-}(i)} v_{j}^{k+1}+\sum_{j \in N_{l}^{+}(i)} v_{j}^{k}\right)-\left(J_{21 i} u_{i}^{k+1}+J_{23 i}\right)}{\sum_{l=1}^{2} \frac{\alpha}{h_{l}^{2}}\left|N_{l}(i)\right|+J_{22 i}},
\end{gathered}
$$

where $w \in(0,2)$ is the relaxation term $\left(w=1\right.$ corresponds to the Gauss-Seidel method), and $N_{l}(i)$ denotes the neighbors of pixel $i$ in the direction of axis $l$ ( $l=1$ for $x$-axis, $l=2$ for $y$-axis) belonging to $\Omega$, making

$$
\begin{aligned}
& N_{l}^{+}(i)=\left\{j \in N_{l}(i) \mid j>i\right\}, \\
& N_{l}^{-}(i)=\left\{j \in N_{l}(i) \mid j<i\right\} .
\end{aligned}
$$

Given the definition of $M, N_{l}^{+}(i)$ represents two pixels: the right neighbor, and the lower pixel. Similarly, $N_{l}^{-}(i)$ are also two pixels: the left neighbor, and the upper pixel. $|N(i)|=4$ for this case.

\subsection{Pointwise-Coupled Gauss-Seidel Scheme}

A variant of the SOR scheme was proposed by Bruhn et al. [2, 7], aimed to improve its convergence rate for real-time implementation, called Pointwise-Coupled Gauss-Seidel. In this scheme, a coupled $2 \times 2$ system is solved at each pixel, and a synchronous update of the OF values for each pixel is performed. Starting from equations (16)-(17), and defining the vector $q_{i}^{k+1}=\left(u_{i}^{k+1}, v_{i}^{k+1}\right)$ at pixel $i$, the system can be written as:

$$
M_{i} q_{i}^{k+1}=g_{i}^{k+1 / 2}
$$

with

$$
M_{i}=\left[\begin{array}{cc}
\sum_{l=1}^{2} \frac{\alpha}{h_{l}^{2}}\left|N_{l}(i)\right|+J_{11 i} & J_{12 i} \\
J_{21 i} & \sum_{l=1}^{2} \frac{\alpha}{h_{l}^{2}}\left|N_{l}(i)\right|+J_{22 i}
\end{array}\right],
$$


and

$$
g_{i}^{k+1 / 2}=\left[\begin{array}{l}
\sum_{l=1}^{2} \frac{\alpha}{h_{l}^{2}}\left(\sum_{j \in N_{l}^{-}(i)} u_{j}^{k+1}+\sum_{j \in N_{l}^{+}(i)} u_{j}^{k}\right)-J_{13 i} \\
\sum_{l=1}^{2} \frac{\alpha}{h_{l}^{2}}\left(\sum_{j \in N_{l}^{-}(i)} v_{j}^{k+1}+\sum_{j \in N_{l}^{+}(i)} v_{j}^{k}+\right)-J_{23 i}
\end{array}\right] .
$$

From Equation (22) it can be shown that an iterative solution can be found by solving the following $2 \times 2$ linear system (here using Cramer's rule):

$$
\begin{aligned}
& u_{i}^{k+1}=\frac{1}{\operatorname{det}\left(M_{i}\right)} \operatorname{det}\left(\left[\begin{array}{lc}
\sum_{l=1}^{2} \frac{\alpha}{h_{l}^{2}}\left(\sum_{j \in N_{l}^{-}(i)} u_{j}^{k+1}+\sum_{j \in N_{l}^{+}(i)} u_{j}^{k}\right)-J_{13 i} & J_{12 i} \\
\sum_{l=1}^{2} \frac{\alpha}{h_{l}^{2}}\left(\sum_{j \in N_{l}^{-}(i)} v_{j}^{k+1}+\sum_{j \in N_{l}^{+}(i)} v_{j}^{k}+\right)-J_{23 i} \sum_{l=1}^{2} \frac{\alpha}{h_{l}^{2}}\left|N_{l}(i)\right|+J_{22 i}
\end{array}\right]\right), \\
& v_{i}^{k+1}=\frac{1}{\operatorname{det}\left(M_{i}\right)} \operatorname{det}\left(\left[\begin{array}{cc}
\sum_{l=1}^{2} \frac{\alpha}{h_{l}^{2}}\left|N_{l}(i)\right|+J_{11 i} & \sum_{l=1}^{2} \frac{\alpha}{h_{l}^{2}}\left(\sum_{j \in N_{l}^{-}(i)} u_{j}^{k+1}+\sum_{j \in N_{l}^{+}(i)} u_{j}^{k}\right)-J_{13 i} \\
J_{21 i} & \sum_{l=1}^{2} \frac{\alpha}{h_{l}^{2}}\left(\sum_{j \in N_{l}^{-}(i)} v_{j}^{k+1}+\sum_{j \in N_{l}^{+}(i)} v_{j}^{k}+\right)-J_{23 i}
\end{array}\right]\right) \text {. }
\end{aligned}
$$

Although the computations for $u_{i}^{k+1}$ and $v_{i}^{k+1}$ can be performed sequentially (i.e. one iteration loop for $u$, then another for $v$ ), the alternating computation for both can prevent problems for small $\alpha$ values that make the term $q^{T} J_{\rho}\left(\nabla_{3} f\right) q$ dominate in the solution. This scheme has been also described with other solver approaches such as multigrid CLG-OF [2].

\section{Algorithm}

This section presents the MS CLG-OF implementation as follows: algorithms 1-2 depict the main algorithm pseudo-code; constants and function descriptions are given as a complement to the source code documentation; finally, a time complexity analysis of the algorithm is presented.

\subsection{Constants}

- $J R O W S=3$ Number of rows of the $J_{\rho}$ matrix associated to a given image position (pixel).

- $J C O L S=3$ Number of columns of the $J_{\rho}$ matrix associated to a given image pixel.

- MIN_GAUSSIAN_SIZE = 3 Minimum Gaussian kernel size allowed for smoothing images/frames. If a computed kernel size (for a given $\rho$ or $\sigma$ ) is lower than this value, no smoothing is performed.

- $E P S=1 \cdot 10^{-12}$ Precision threshold (epsilon) value for the numerical relaxation computations.

- MIN_ERROR $=1 \cdot 10^{-4}$ Convergence threshold for the main iteration loop. It is compared to the mean variation of the OF vectors between iterations (the mean variation value must be lower). At the iteration $k$, for each pixel $i$ ( $N$ total), the variation is computed as $\sqrt{\frac{\sum_{i}\left(u_{i}^{k}-u_{i}^{k-1}\right)^{2}+\left(v_{i}^{k}-v_{i}^{k-1}\right)^{2}}{N}}$. 


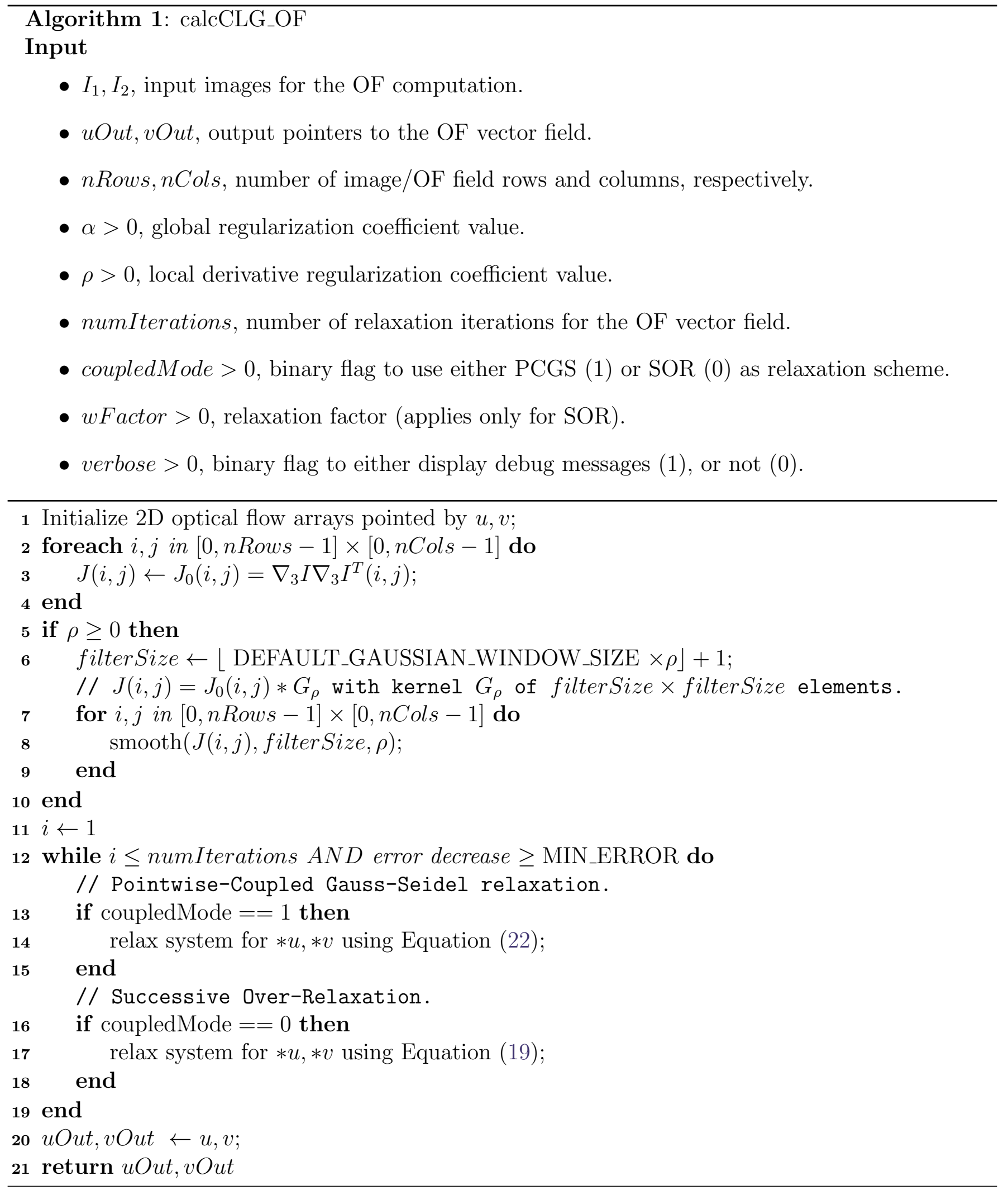



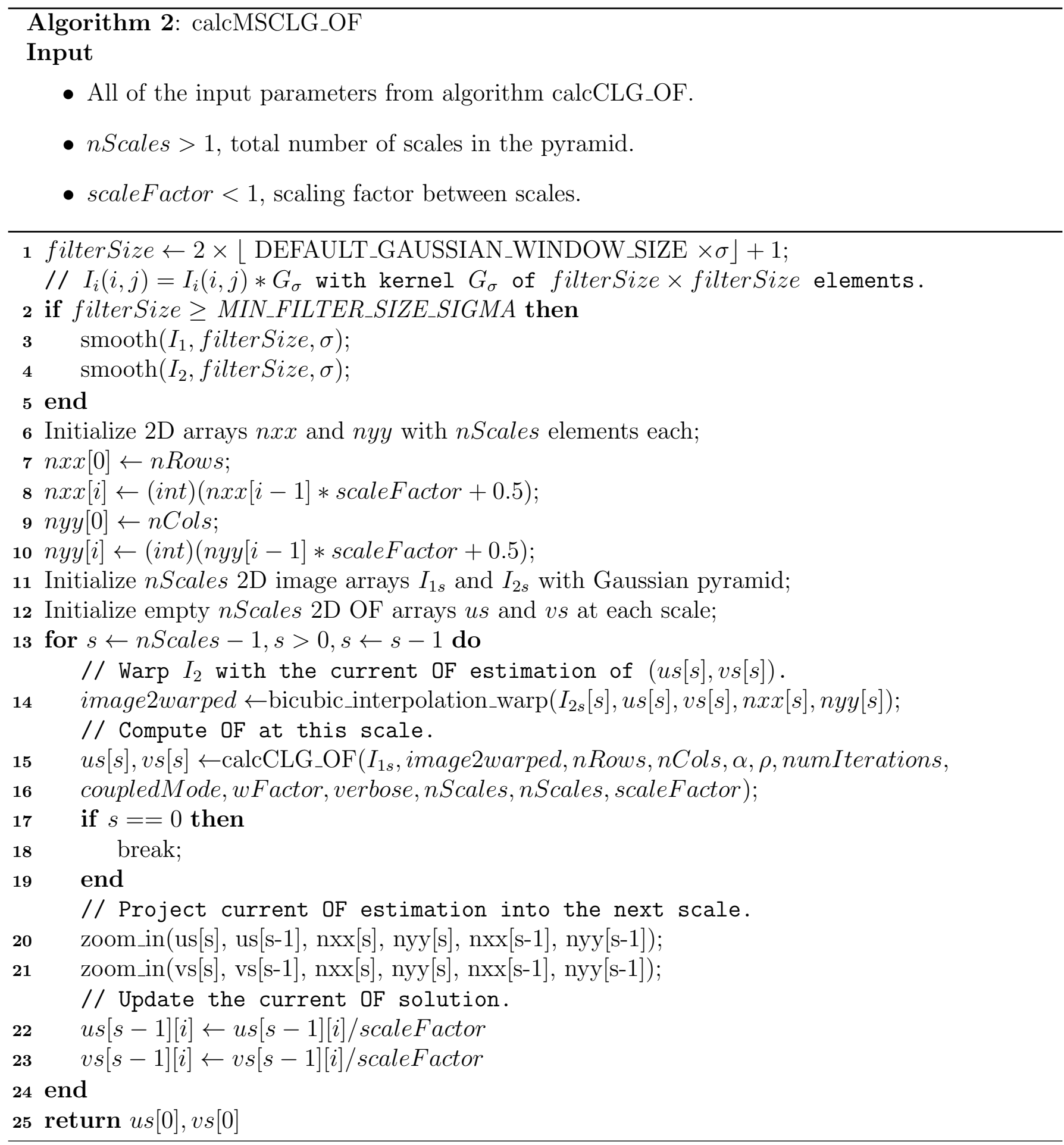


\subsection{Function Description}

This section summarizes the purpose and parameters of the implemented functions. For input/output and MS functions the implementation contributed by Meinhardt-Llopis et al. [11] is used. Pointer variables to 1 - and 2-dimensional arrays are denoted with the prefixes ${ }^{*}$ and ${ }^{* *}$, respectively.

- calcCLG_OF (*image1, *image2, *uOut, *vOut, nCols, nRows, iterations, alpha, rho, wFactor, verbose, coupledMode)

CLG-OF computation function implements Algorithm 1. image1 and image 2 point to the input images for the OF (in the form of $1 \mathrm{D}$ arrays of $n$ Rows $\times n$ Cols elements each). uOut, vOut are output pointer variables for the vertical and horizontal OF components, of $n$ Cols $\times n$ Rows elements each. iterations, alpha and rho give the input values for maximum number of iterations, global smoothing coefficient $\alpha$, and local spatio-temporal smoothing coefficient $\rho$, respectively. The flag coupledMode sets the iteration mode to SOR (0) or PCGS (1). When using SOR, wFactor $\in(0,2)$ is the relaxation factor. The verbose flag enables verbose output.

- calcMSCLG_OF(*image1, *image 2, *uOut, *vOut, nCols, nRows, iterations, alpha, rho, sigma, wFactor, nScales, scaleFactor, coupledMode, verbose)

MS CLG-OF computation function implements Algorithm 2, by calling calcCLG_OF with the corresponding input parameters (which have the same names). sigma is the Gaussian image smoothing parameter. scaleFactor $\in(0,1)$ is the pyramid resizing factor, e.g. scale Factor $=$ 0.5 accounts for half size reductions. $n$ Scales gives the number of scales, provided that the final size of the coarsest pyramid level is large enough to compute the derivatives (if not, the maximum scale number is computed and used). The verbose flag enables verbose output.

- SOR_at $(* * u, * * v, * * J, i, j$, alpha, wFactor $)$

Solves the CLG-OF equations for the $u, v$ components at image pixel $[i, j]$, according to Equation (19). alpha gives the value of the global smoothing coefficient. $J$ points to the arrays with the computed (and possibly smoothed) derivatives. wFactor $\in(0,2)$ is the relaxation factor.

- $\operatorname{relax} S O R(* * u, * * v, * * J, n R o w s, n$ Cols, alpha, wFactor $)$

SOR iteration for solving the CLG-OF equations by calling the function $S O R \_a t$ for each pixel (with equally named input parameters), and Equation (10) for boundary conditions. The horizontal and vertical OF components are $u, v$ of $n$ Rows $\times n$ Cols elements each.

- relaxPointwiseCoupledGaussSeidel $(* * u, * * v, * * J$, nRows, $n$ Cols, alpha)

PCGS relaxation iteration for solving the CLG-OF equations. Updates the value of the OF vector field components $u, v$ according to equations (23)-(24), using Cramer's rule. If the discriminant value is lower than EPS a SOR iteration is performed instead. Equation (10) is used for boundary conditions. Function parameters are the same described for calcCLG_OF and SOR_at.

- boundaryCondition $(* * u, * * v, n$ Rows, $n$ Cols $)$

Performs SOR and PCGS iterations at the boundary pixels of $u, v$ (rows 0 and $n$ Rows -1 , columns 0 and $n$ Cols -1 ), by copying values from the non-boundary neighbor pixels.

\subsubsection{Utility functions}

- computeDerivatives $(* *$ image $1, * *$ image $2, * * d f d x, * * d f x y, n$ Rows, $n$ Cols $)$ Computes the discrete spatial derivatives for the input time frames image1, image 2 of $n$ Rows $\times$ $n C o l s$ elements. The derivatives are stored in the arrays pointed by $d f d x, d f d y$ ( $x, y$ coordinates) 
of $n$ Rows $\times n$ Cols elements each. The derivatives are computed by using a $3 \times 1$ stencil with values $[-0.5,0.0,+0.5]$ (centered differences).

- computeJTensor $(* * d f d x, * * d f d y, * * d f d t, * * J, n R o w s, n C o l s)$

Computes and stores the values of the tensor matrix $J$ at each image pixel. $d f d x, d f d y, d f d t$ point to the derivative matrices of the input images, containing $n$ Rows $\times n$ Cols elements each. The result is pointed by $J$, with $n$ Rows $\times n$ Cols $\times J R O W S \times J C O L S$ elements. For a given pixel $[i, j]$ the associated tensor is a $3 \times 3$ matrix, with elements

$$
\begin{aligned}
& J[1][1][i][j]=I_{x}[i][j] * I_{x}[i][j] \\
& J[1][2][i][j]=I_{x}[i][j] * I_{y}[i][j] \\
& J[1][3][i][j]=I_{x}[i][j] * I_{t}[i][j] \\
& J[2][2][i][j]=I_{y}[i][j] * I_{y}[i][j] \\
& J[2][3][i][j]=I_{y}[i][j] * I_{t}[i][j] \\
& J[3][3][i][j]=I_{t}[i][j] * I_{t}[i][j],
\end{aligned}
$$

being symmetric with respect to its diagonal, so only the upper triangular part is stored.

- matrixSmooth(** matrix, nRows, nCols, kernelSigma)

Performs a Gaussian smoothing over a given input matrix of $n$ Rows $\times n$ Cols elements, by applying a square kernel parametrized by its standard deviation kernelSigma. It is called from calcCLG_OF for smoothing the input images and the derivatives matrix, according to the values of sigma and rho, respectively. The kernel size is computed in a way that ensures odd values (i.e. symmetric Gaussian kernels with a single central value as maximum), greater than MIN_GAUSSIAN_SIZE.

- $\operatorname{correctIndex}(p$, size $)$

Given an index $p$, returns $p$ if $p \in[0$, size -1$]$. If not, returns its closest number from [0, size-1].

- $\operatorname{lin} 2 b y 2 \operatorname{det}(a, b, c, d)$

Computes and return the determinant of a given $2 \times 2$ matrix, $A=\left[\begin{array}{ll}a & b \\ c & d\end{array}\right]$. The determinant $D$ is computed as $D=a d-b c$.

- $p$ Matrix(nRows, nCols)

Initializes and returns a 2D pointer array of a given number of rows and columns, nRows and $n$ Cols respectively.

- freePmatrix $(* *$ mat, $n$ Rows)

Deallocates a given 2D pointer matrix mat, given as a pointer to an array of nRows pointers.

- $x$ malloc(size)

Array memory allocation function, for a given memory size. 


\subsection{Time Complexity}

Assume a square image of $N_{s}$ pixels at each pyramid level $s\left(N_{0}\right.$ is the original image level), and filter kernel sizes up to $k<<N_{s}$ (for smoothing and derivatives). The overall cost is the sum of the cost of building the pyramid by filtering operations $O\left(N_{s} k\right)$ each, plus the OF iterations at each pyramid level. OF iterations by matrix inversion can be computed in $O\left(N_{s}^{2}\right)$ time, but iterative methods such as SOR and PCGS perform closer to $O\left(C N_{s}\right)$, where $C$ depends on the input. However, $C<<N_{0}$, for instance $C<3000$ for the Middlebury dataset (Table 2). Thus, as the cost is dominated by the finer scale (of size $N_{0}$ ), the running time of the MS CLG-OF algorithm is $O\left(C N_{0}\right)$.

\section{Evaluation}

Here we present examples of the implemented CLG-OF applied to different image sequences. First, in Subsection 4.1 we illustrate the effect of different values for the regularization coefficients $\alpha$ and $\rho$ in the OF fields. Next, in Subsection 4.2 we present and briefly discuss results from synthetic sequences with known ground-truth OF, comparing the MS CLG-OF estimation error against examples from the original CLF-OF article and the Middlebury database.

\subsection{Example Sequences}

The first example, shown in Figure 1, is the CLG-OF field for a model structure in fluorescence microscopy, corresponding to a moving point signal (fluorescent protein, a-b). The signal was convolved with a theoretical point spread function (PSF, b-c) of a confocal microscope (a PSF acts as a smoothing function that blurs signals). See details of simulation and tests for optimal parameters in the work of Delpiano et al. [5]. Next, figures 2-5 illustrate the effects of different values for $\alpha$ and $\rho$ in the resulting $\mathrm{OF}$ fields.
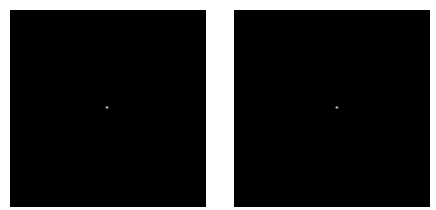

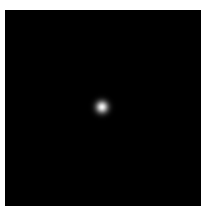

a

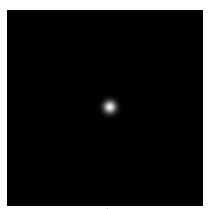

b

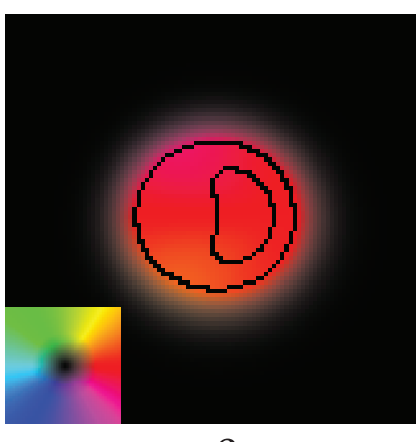

$\mathrm{C}$

Figure 1: CLG-OF for a moving point source, simulating a fluorescent signal in a confocal microscopy image (adapted from Delpiano et al. [5]). a,b: time frames of a synthetic point signal (top), and their computed confocal PSF-convolved images (down), of $100 \times 100$ pixels each, for a confocal microscope with $60 \mathrm{x}$ water objective and wavelengths of $543 / 560 \mathrm{~nm}$ for excitation/emission. Pixel size is $107 \mathrm{~nm}$ in $x$ and $y$. The point displacement between the two frames is 3 pixels $(321 \mathrm{~nm})$. c: CLG-OF field of the PSF-convolved images, computed with parameter values: $\alpha=200, \rho=5, \sigma=0$, nScales $=1$, iterations $=200$, using PCGS solver. The OF vector color code is shown at the bottom left image. 


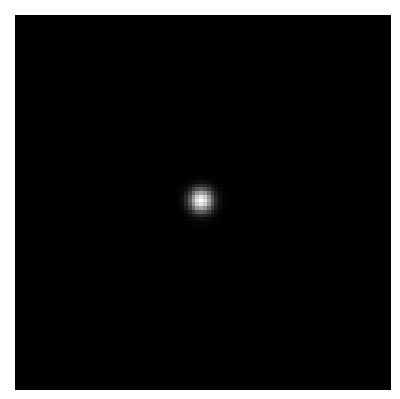

$\mathrm{I}_{1}$ convolved

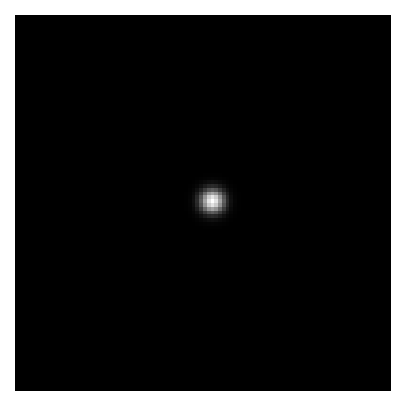

$\mathrm{I}_{2}$ convolved

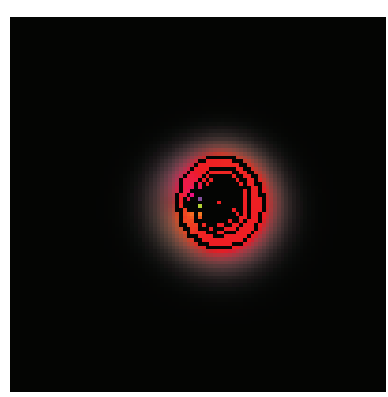

$\alpha=20, \rho=0$

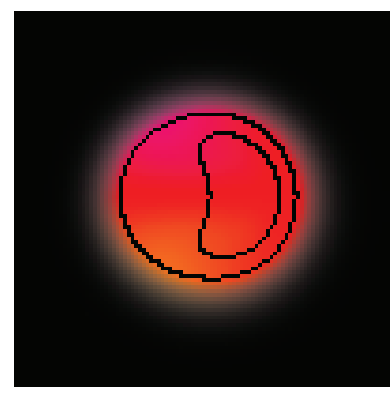

$$
\alpha=20, \rho=5
$$

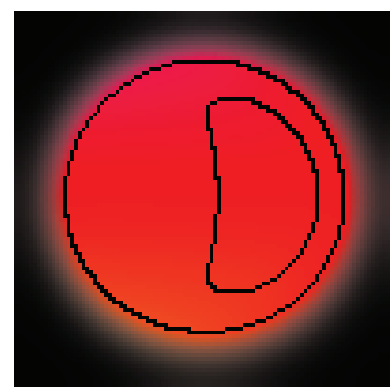

$$
\alpha=20, \rho=10
$$

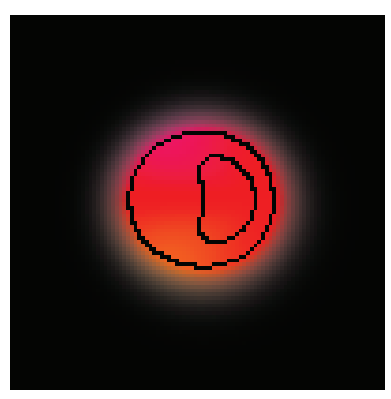

$$
\alpha=200, \rho=0
$$

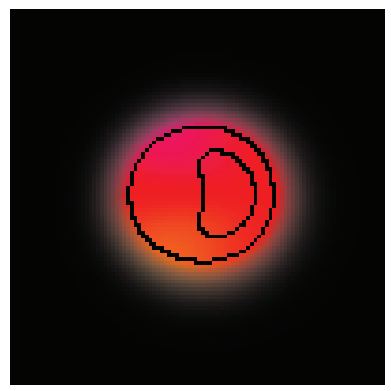

$$
\alpha=200, \rho=5
$$

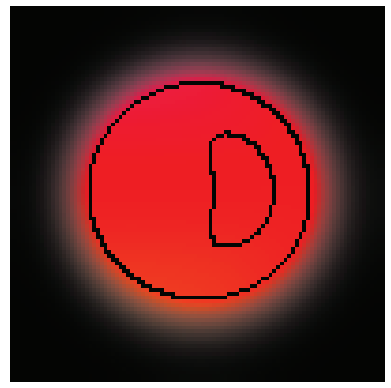

$$
\alpha=200, \rho=10
$$

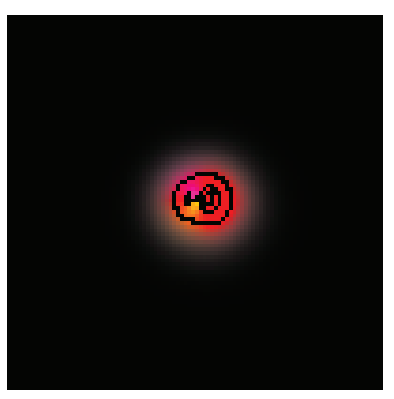

$\alpha=2000, \rho=0$

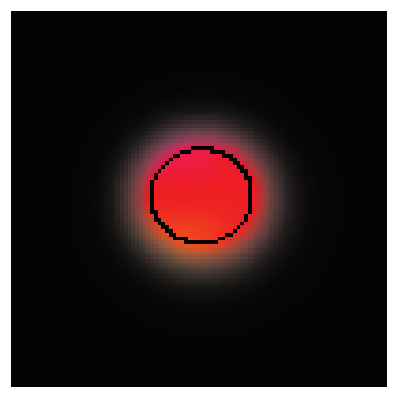

$$
\alpha=2000, \rho=5
$$

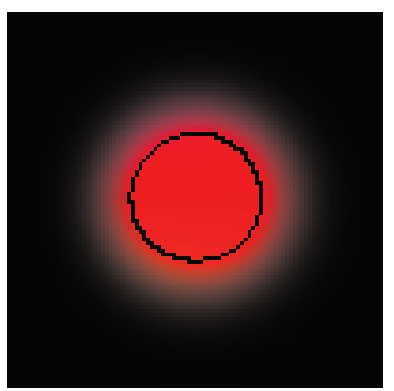

$\alpha=2000, \rho=10$

Figure 2: Different parameter values for CLG-OF and their corresponding OF fields for the synthetic point source fluorescence image from Figure1, with an horizontal displacement of three pixels. $I_{1}, I_{2}$ are the input images. Computed with parameter values: $\sigma=0, n$ Scales $=1$, iterations $=200$, using PCGS solver. Vectors are color coded as in Figure 1.c. 

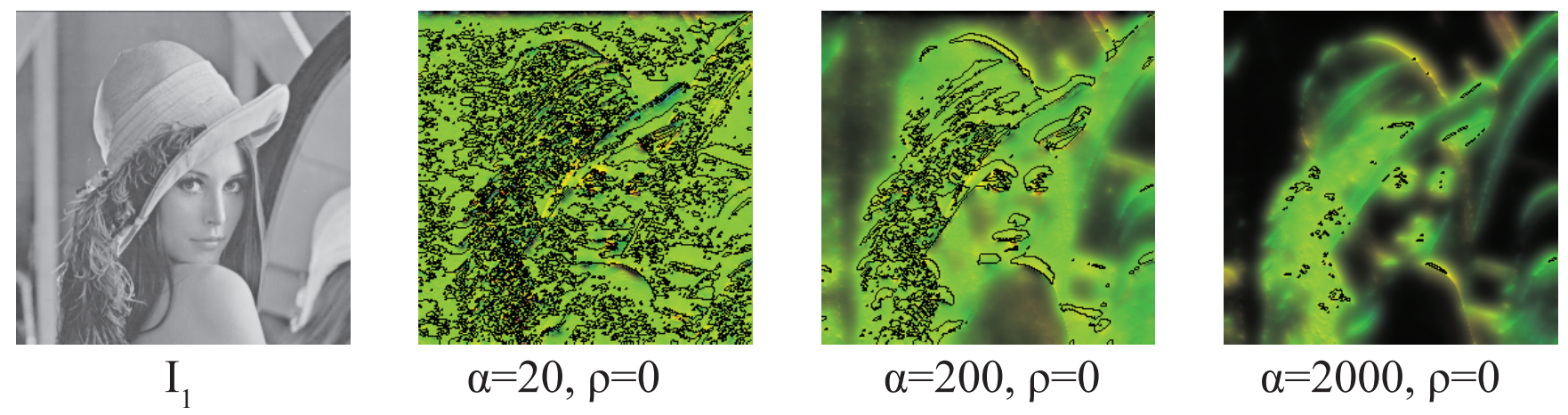

$$
\alpha=20, \rho=0
$$
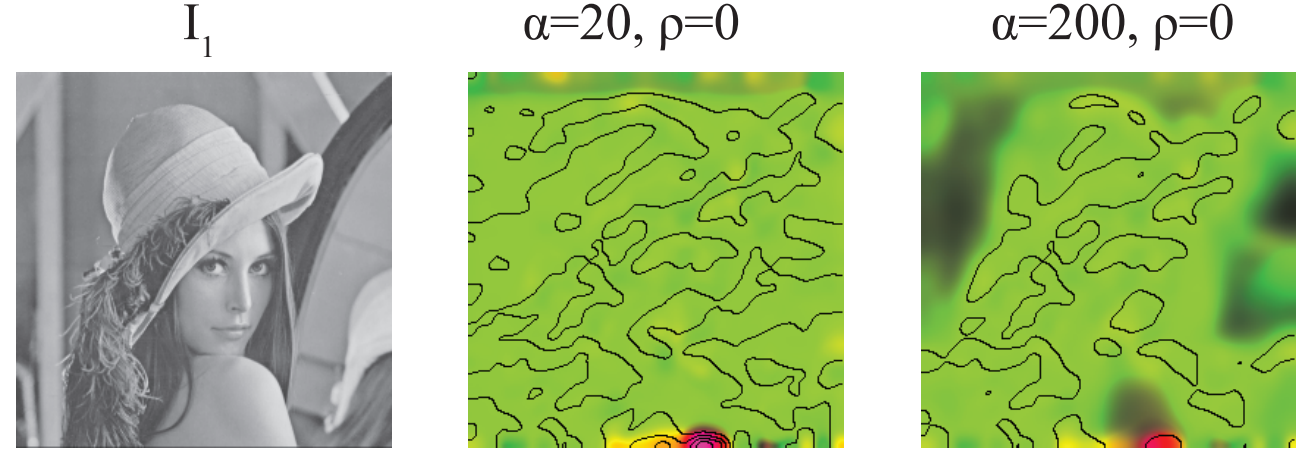

$$
\alpha=2000, \rho=0
$$
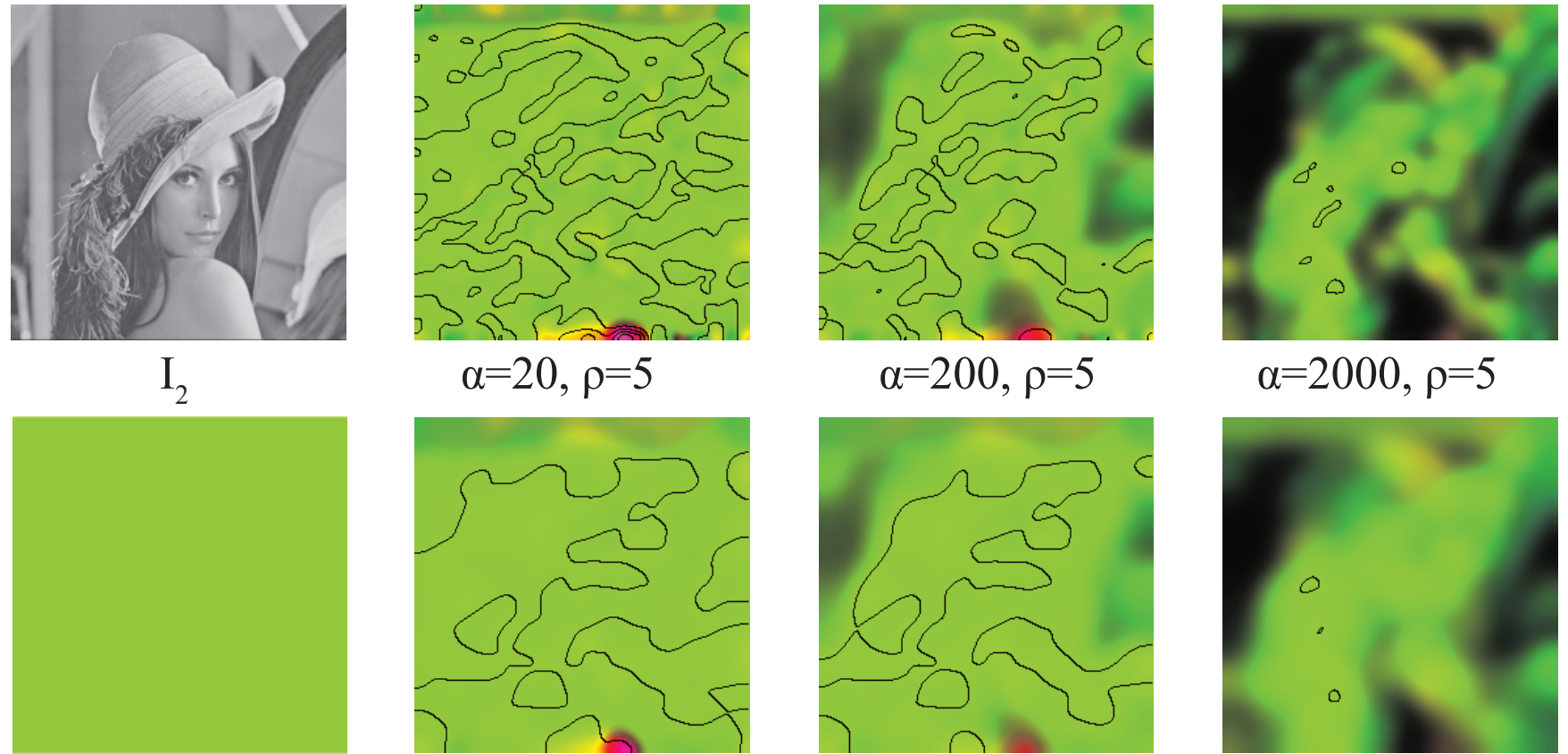

$$
\alpha=20, \rho=5
$$

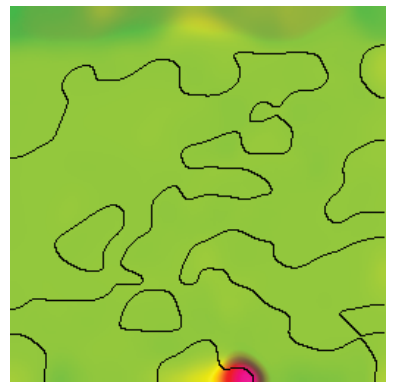

True flow

$$
\alpha=20, \rho=10
$$

$$
\alpha=200, \rho=5
$$

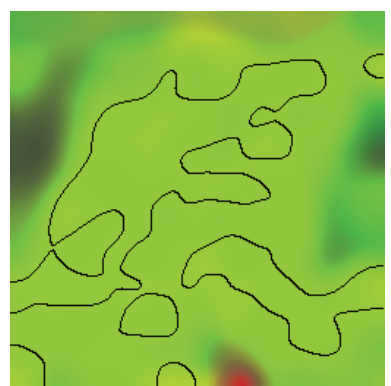

$$
\alpha=200, \rho=10
$$

$$
\alpha=2000, \rho=5
$$

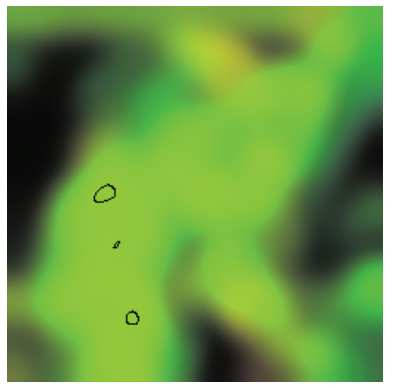

$\alpha=2000, \rho=10$

Figure 3: Different parameter values for CLG-OF and their corresponding OF fields for the Lena image $(256 \times 256$ pixels, gray-scale version $)$, with an horizontal displacement of one pixel. $I_{1}, I_{2}$ are the input images. Computed with parameter values: $\sigma=0, n$ Scales $=1$, iterations $=200$, using PCGS solver. Vectors are color coded as in Figure 1.c. 

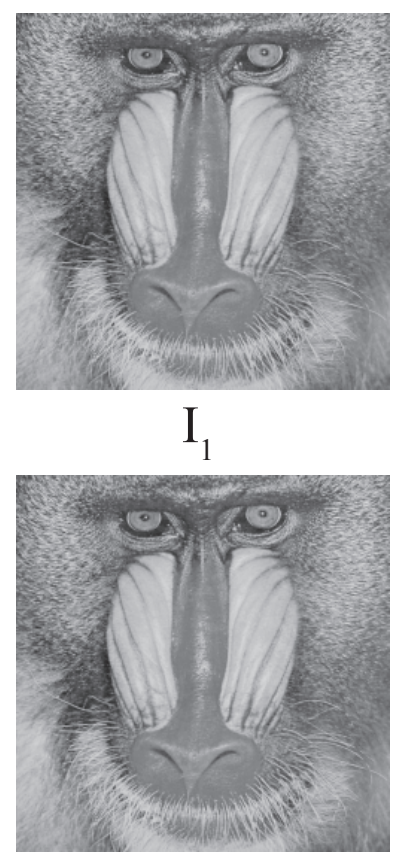

$\mathrm{I}_{2}$

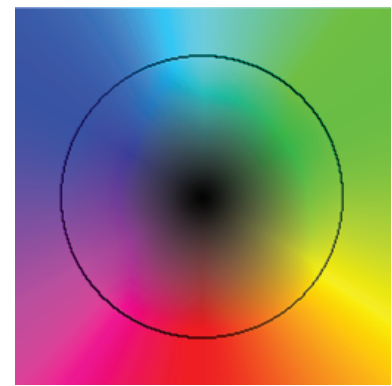

True flow

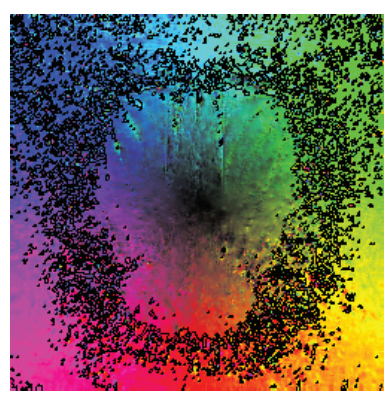

$\alpha=20, \rho=0$

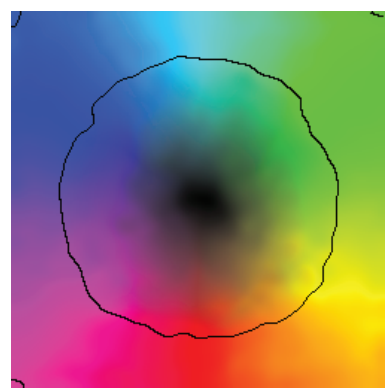

$$
\alpha=20, \rho=5
$$

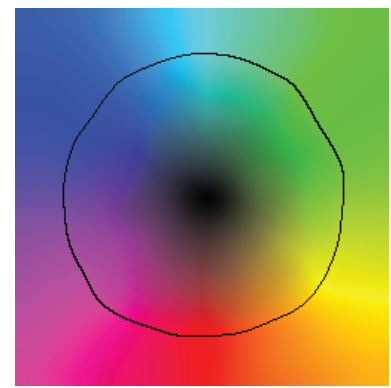

$\alpha=20, \rho=10$

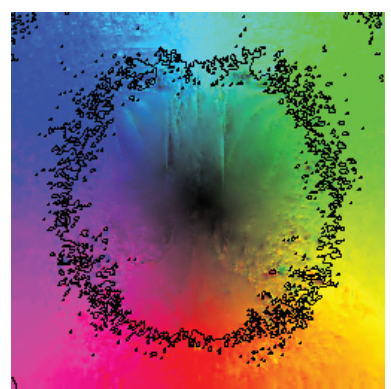

$\alpha=200, \rho=0$

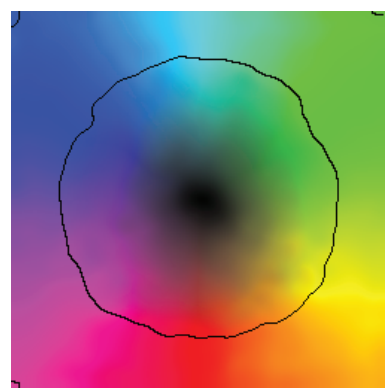

$$
\alpha=200, \rho=5
$$

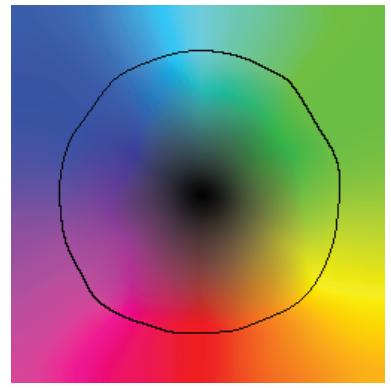

$\alpha=200, \rho=10$

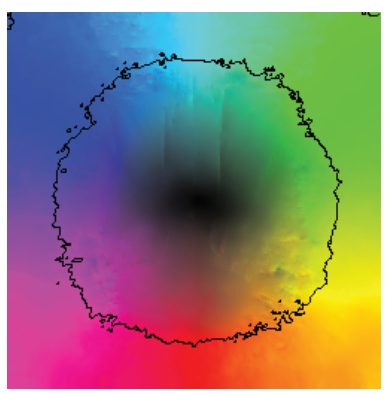

$\alpha=2000, \rho=0$

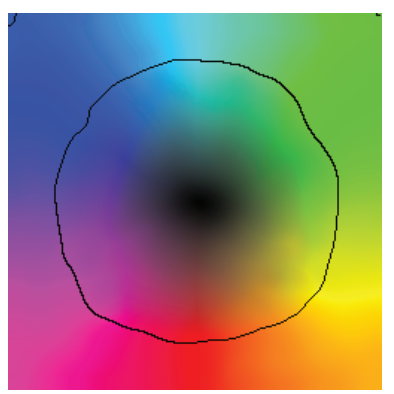

$$
\alpha=2000, \rho=5
$$

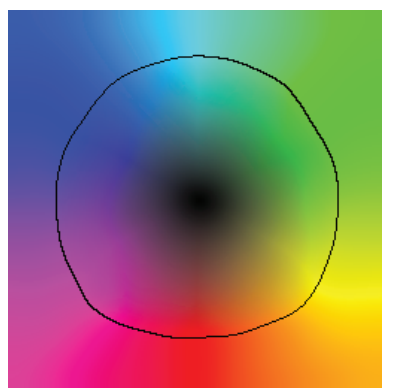

$\alpha=2000, \rho=10$

Figure 4: Different parameter values for MS CLG-OF and their corresponding OF fields for the baboon rotation $(256 \times 256$ pixels, gray-scale version $)$ images. $I_{1}, I_{2}$ are the input images. Computed with parameter values: $\sigma=0, n$ Scales $=3$, scaleFactor $=0.65$, iterations $=200$, using PCGS solver. Vectors are color coded as in Figure 1.c. 

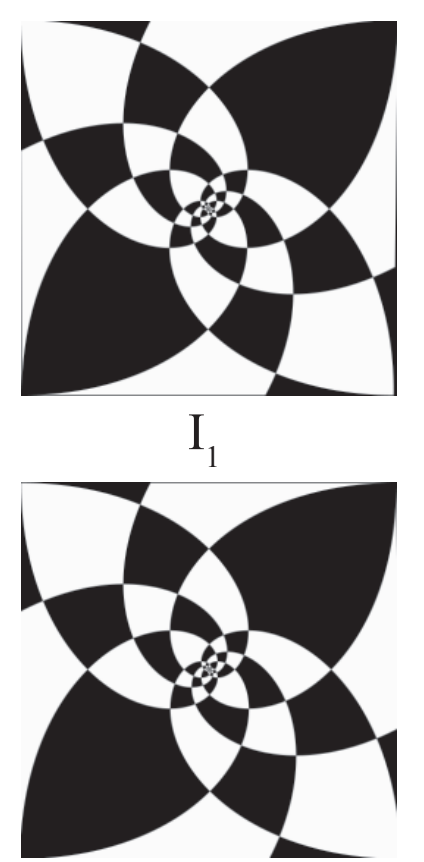

$\mathrm{I}_{2}$

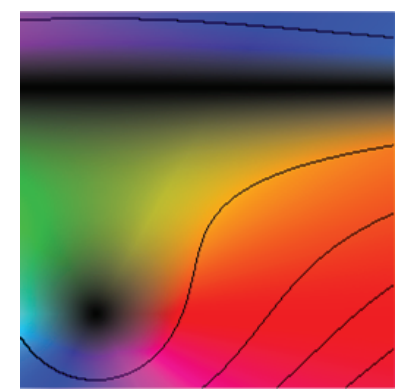

True flow

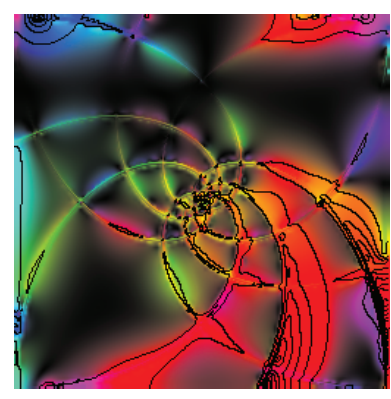

$$
\alpha=20, \rho=0
$$

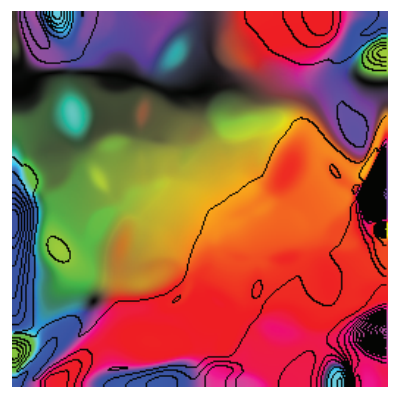

$$
\alpha=20, \rho=5
$$

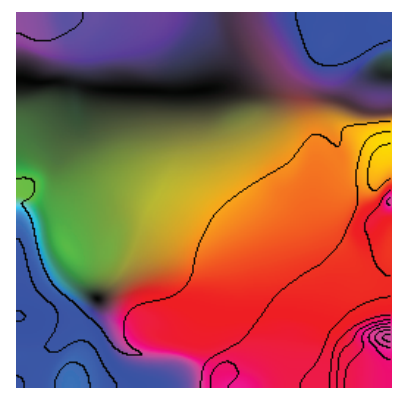

$\alpha=20, \rho=10$

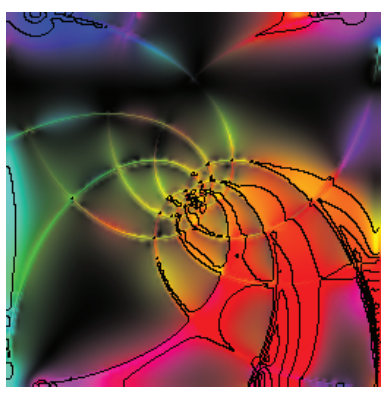

$$
\alpha=200, \rho=0
$$

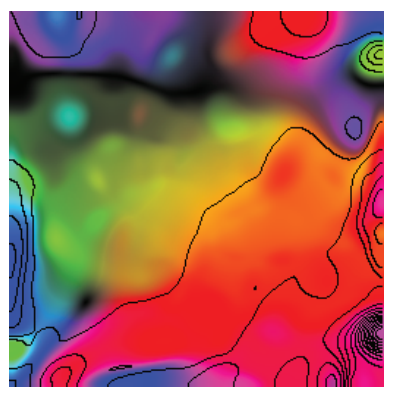

$$
\alpha=200, \rho=5
$$

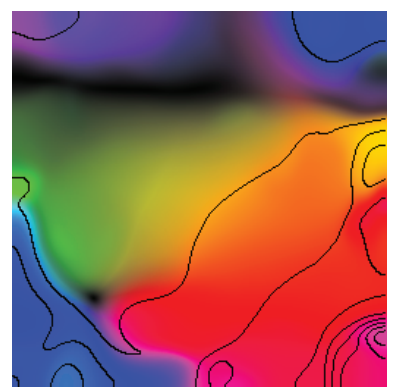

$$
\alpha=200, \rho=10
$$

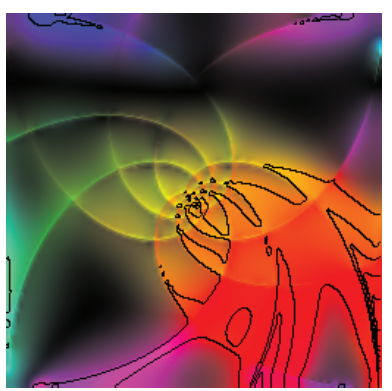

$$
\alpha=2000, \rho=0
$$

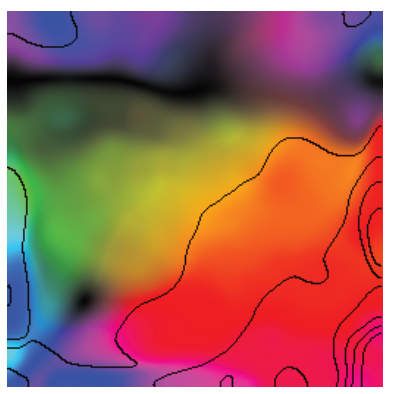

$$
\alpha=2000, \rho=5
$$

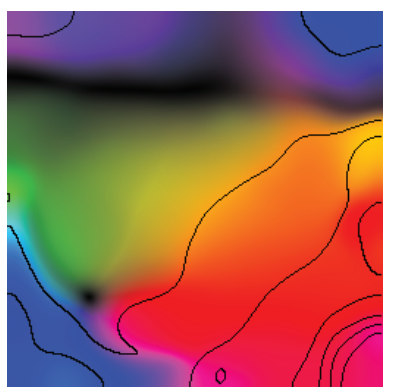

$\alpha=2000, \rho=10$

Figure 5: Different parameter values for MS CLG-OF and their corresponding OF fields for the spiral image with an homography transformation. $I_{1}, I_{2}$ are the input images. Computed with parameter values: $\sigma=0, n$ Scales $=3$, scaleFactor $=0.65$, iterations $=200$, using PCGS solver. Vectors are color coded as in Figure 1.c. 


\subsection{Error Metrics}

We tested the MS CLG-OF implementation with image sequences from Bruhn et al. [4], shown in Figure 6, and from the Middlebury database [1], shown in Figure 7. For both cases, the Average Angular Error (AAE) and Average Endpoint Error (AEE) against ground-truth OF fields, as defined by Baker et al. [1], were measured. We also measured computation times, using a PC workstation with a $3.4 \mathrm{GHz}$ Intel Core i7 4930K CPU and $64 \mathrm{~GB}$ of RAM, running the 64-bit Ubuntu Linux 12.04 operating system. The results are summarized in tables 1 and 2 .

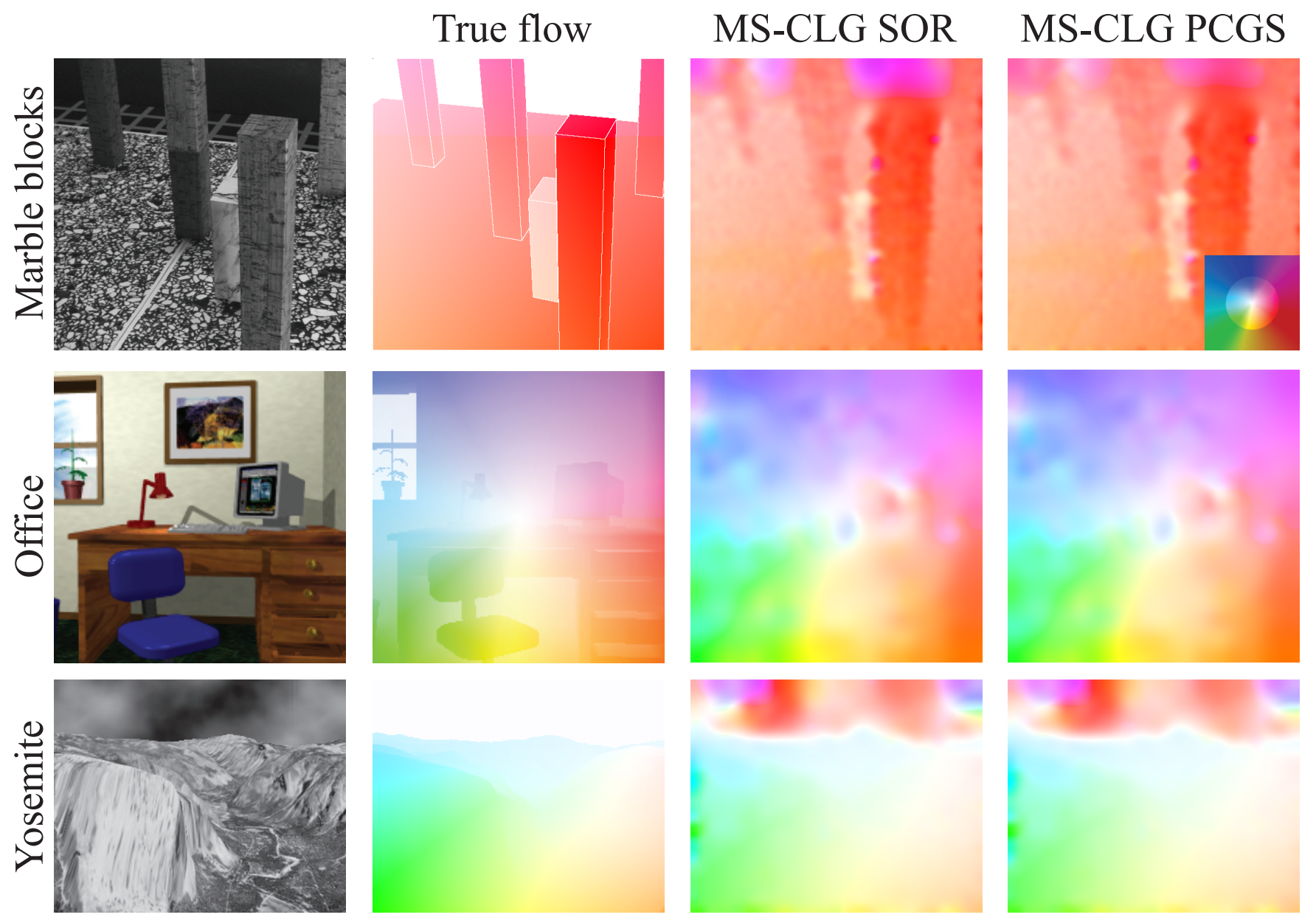

Figure 6: Results of the MS CLG-OF implementation with the SOR and PCGS numerical schemes, in selected images from the original GLG-OF article [4]. Computed with parameter values: $\alpha=200, \rho=$ $5.0, \sigma=0.85$, scaleFactor $=0.65$, MIN_ERROR $=0.0001$, iterations $=10000$, and $w$ Factor $=1.8$ (SOR only). The number of scales $n$ Scales was dependent in the image size, thus chosen as 7, 5, and 6 respectively. Convergence was achieved before the indicated number of iterations, counted at the original image level, as shown in Table 1. The vector color code is shown at the top right image.

\subsection{Discussion}

As can be seen in Table 1, our implementation performs worse than the original work by Bruhn et al. [4] (10\% - 90\% worse). Unfortunately, AEE values and the exact images/measurements are not available (the sequences have more than two images), so an exact comparison could not be made. Within our tests, we found that for a similar error margin, the PCGS iterative scheme performs 2 to 10 times faster than SOR, but with slightly worse error metrics. 

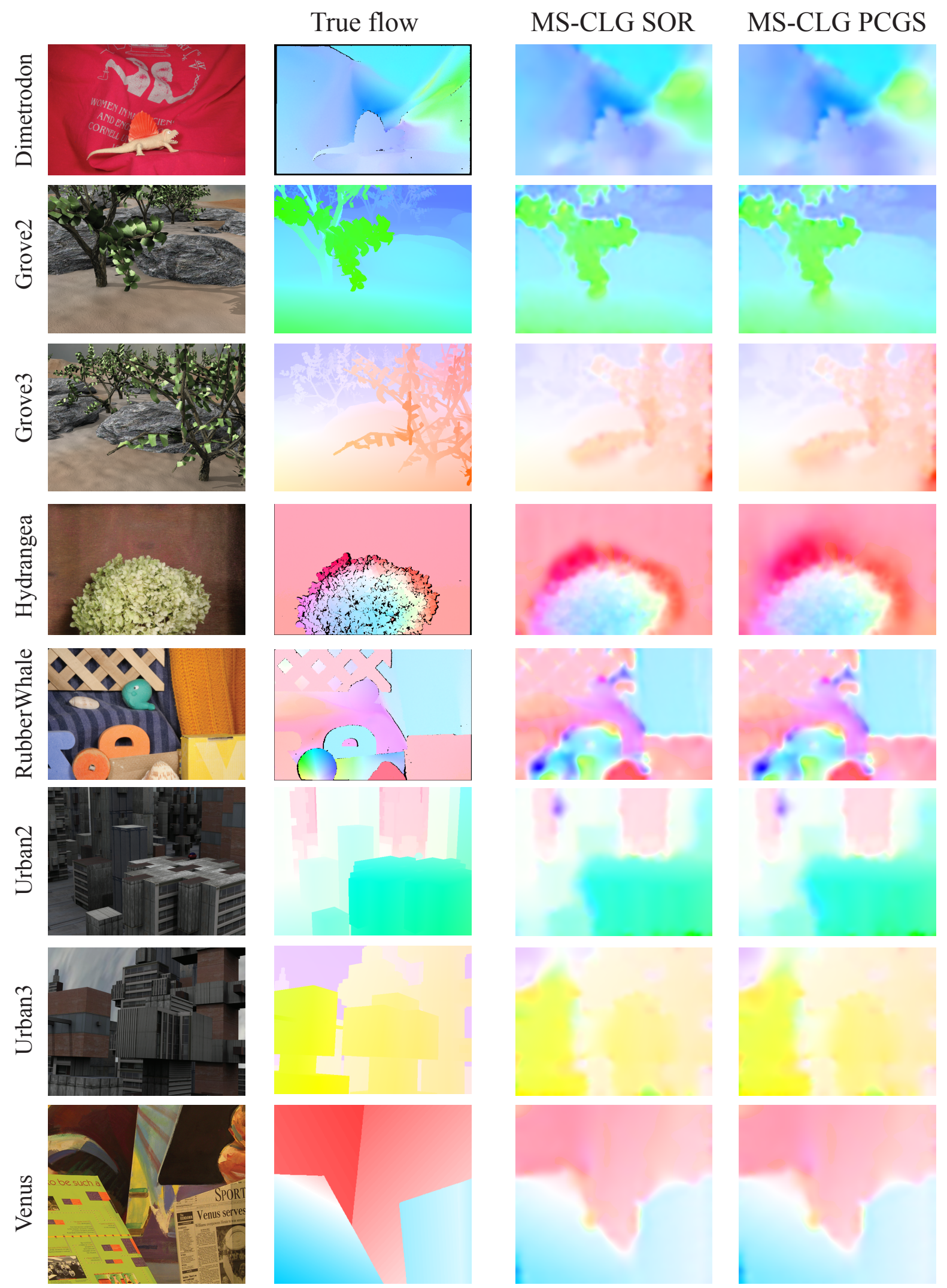

Figure 7: Results of the MS CLG-OF implementation with the SOR and PCGS numerical schemes, in selected images from the Middlebury dataset [1]. Computed with parameter values: $\alpha=200$, $\rho=5.0, \sigma=0.85, n$ Scales $=7$, scaleFactor $=0.65$, MIN_ERROR $=0.0001$, iterations $=10000$, and $w$ Factor $=1.8$ (SOR only). Convergence was achieved before the indicated number of iterations, counted at the original image level, as shown in Table 2. Vectors are color coded as in Figure 6. 


\begin{tabular}{lrrr}
\hline & Marble blocks & Office & Yosemite \\
\hline SOR & & & \\
\hline AAE $\left(^{\circ}\right)$ & $\mathbf{9 . 9 9}$ & $\mathbf{4 . 7 1}$ & $\mathbf{3 . 1 1}$ \\
AEE & 0.30 & 0.10 & 0.17 \\
Iterations & 875 & 2017 & 2118 \\
Time $[\mathrm{s}]$ & 17.01 & 5.42 & 11.51 \\
\hline PCGS & & & \\
\hline AAE $\left(^{\circ}\right)$ & $\mathbf{9 . 9 9}$ & $\mathbf{4 . 8 2}$ & $\mathbf{3 . 0 9}$ \\
AEE & 0.30 & 0.10 & 0.17 \\
Iterations & 190 & 276 & 1316 \\
Time $[\mathrm{s}]$ & 9.19 & 1.7 & 6.51 \\
\hline SOR by Bruhn et al. $[4]$ & & \\
\hline AAE $\left(^{\circ}\right)$ & $\mathbf{5 . 3 0}$ & $\mathbf{4 . 3 3}$ & $\mathbf{2 . 6 4}$ \\
\hline
\end{tabular}

Table 1: Comparison of AAE and AEE measurements of the implemented MS CLG-OF with the SOR and PCGS numerical schemes, against the original reported results by Bruhn et al. [4] for the images shown in Figure 6. Available ground-truth was taken into account at pixels with OF vector magnitude less than 1000 in Marble blocks and Office sequences, and other than 0.0406 (clouds displacement) for Yosemite sequence. From Marble blocks and Office the first two frames were used.

\begin{tabular}{|c|c|c|c|c|c|c|c|c|}
\hline & Dimetro. & Grove2 & Grove3 & Hydrangea & RubberWhale & Urban2 & Urban3 & Venus \\
\hline \multicolumn{9}{|c|}{ MS CLG SOR } \\
\hline $\operatorname{AAE}\left({ }^{\circ}\right)$ & 4.3 & 4.56 & 9.79 & 4.09 & 11.94 & 7.66 & 15.51 & 10.73 \\
\hline $\mathrm{AEE}$ & 0.22 & 0.31 & 1.31 & 0.6 & 0.37 & 1.0 & 1.65 & 0.65 \\
\hline Iterations & 589 & 1713 & 1118 & 2772 & 814 & 3443 & 547 & 1398 \\
\hline Time [s] & 11.26 & 37.75 & 24.9 & 42.57 & 14.25 & 71.42 & 13.52 & 15.46 \\
\hline \multicolumn{9}{|c|}{ MS CLG PCGS } \\
\hline $\operatorname{AAE}\left({ }^{\circ}\right)$ & 7.7 & 4.96 & 10.4 & 6.63 & 12.69 & 8.35 & 18.76 & 11.13 \\
\hline $\mathrm{AEE}$ & 0.37 & 0.34 & 1.44 & 1.16 & 0.39 & 1.13 & 1.92 & 0.68 \\
\hline Iterations & 90 & 113 & 116 & 56 & 207 & 184 & 135 & 216 \\
\hline Time $[\mathrm{s}]$ & 4.52 & 7.17 & 9.15 & 3.38 & 7.01 & 9.5 & 9.05 & 5.58 \\
\hline
\end{tabular}

Table 2: AAE and AEE measurements for the implemented MS CLG-OF with the SOR and PCGS numerical schemes, for the Middlebury database images shown in Figure 7. Available ground-truth was taken into account at pixels with OF vector magnitude less than 1000 for all the sequences. 
Although some parameter values of the present implementation were kept fixed for the examples, it becomes clear that a fine tuning of $\alpha, \rho, n$ Scales, wFactor, and scaleFactor is required for the best results. For instance, by varying the value of $w$ Factor between 1.8 and 1.9, the AAE can be further improved in $1^{\circ}-2^{\circ}$ (not shown). Figures $2-5$ and the variability of the error metrics illustrate this issue, as well as reported results from Delpiano et al. [5], Hubený et al. [9], Meinhardt-Llopis et al. [11] and Sun et al. [13].

We remind that, other than the chosen numerical scheme and parameter values, the use of late linearization (not implemented here) allows for a better handling of larger displacements $\underline{d}=\left[d_{x}, d_{y}\right]$, by making $I_{1}(x, y)-I_{2}\left(x+d_{x}, y+d_{y}\right)=0$ instead of the right side in Equation (1). In order to implement it, two nested iteration loops are required: one for fixed values of $\underline{d}$ iterating over $\underline{V}$, and one for $\underline{d}$ performing multiple warpings at each scale. Significant improvements can be achieved this way, but still a numerical solver such as SOR or PCGS must be used for the $\underline{V}$ loop.

\section{Acknowledgments}

Funding: CONICYT PhD scholarship (JJ), FONDECYT 3140447 (MC) / 1120579 (SH,JJ), FONDEF D11|1096 (SH,JJ), US-LACRN (MC), and ICM P09-015-F (BNI). The authors would like to thank Haldo Spontón and Juan Cardelino for supporting the preparation of this work, and the IPOL editor and reviewers for their valuable insights and suggestions.

\section{Image Credits}

All of the OF field and error measurement images (Figures 1,7) by the authors, except the ground truth ("True flow") images by IPOL.

(Figures 1,2$)$ by the authors.

(Figure 3) from the USC-SIPI Image Database ${ }^{3}$.

(Figure 4) from the USC-SIPI Image Database ${ }^{4}$.

(Figure 5) by Mark Dow, "Logarithmic spirals, waves and tilings" 5.

(Figure 6) from Image Sequence Server (Group Prof. Dr. H.-H. Nagel) at Institut für Algorithmen und Kognitive Systeme, Universität Karlsruhe ${ }^{6}$.

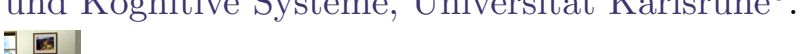

(Figure 6) by Galvin et al. [6].

(Figure 6) by Lynn Quam.
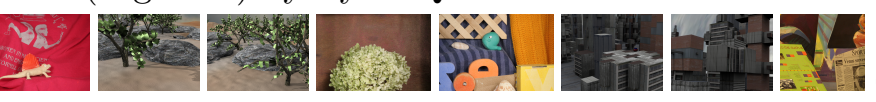

(Figure 7) from the Middlebury benchmark database [1].

\footnotetext{
${ }^{3}$ http://sipi.usc.edu/database/database.php?volume=misc

${ }^{4}$ http://sipi.usc.edu/database/database .php?volume=misc

${ }^{5}$ http://lcni.uoregon.edu/ dow/Geek_art/Logarithmic_spirals/Logarithmic_spirals_waves_tilings . html

${ }^{6}$ http://i21www.ira.uka.de/image_sequences/
} 


\section{References}

[1] S. Baker, D. Scharstein, J.P. Lewis, S. Roth, M.J. Black, and R. Szeliski, $A$ database and evaluation methodology for optical flow, International Journal of Computer Vision, 92 (2011), pp. 1-31. http://dx.doi.org/10.1007/s11263-010-0390-2.

[2] A. Bruhn, J. Weickert, C. Feddern, T. Kohlberger, and C. Schnörr, Variational optical flow computation in real time, Image Processing, IEEE Transactions on, 14 (2005), pp. 608-615. http://dx.doi.org/10.1109/TIP.2005.846018.

[3] A. Bruhn, J. Weickert, And C. Schnörr, Combining the advantages of local and global optic flow methods, in Pattern Recognition, Luc Van Gool, ed., vol. 2449 of Lecture Notes in Computer Science, Springer Berlin Heidelberg, 2002, pp. 454-462. http://dx.doi.org/10. 1007/3-540-45783-6_55.

[4] _ Lucas/Kanade meets Horn/Schunck: Combining local and global optic flow methods, International Journal of Computer Vision, 61 (2005), pp. 211-231. http://dx.doi.org/10.1023/ B\%3AVISI.0000045324.43199.43.

[5] J. Delpiano, J. Jara, J. Scheer, O.A. Ramírez, J. Ruiz-del Solar, and S. Härtel, Performance of optical flow techniques for motion analysis of fluorescent point signals in confocal microscopy, Machine Vision and Applications, 23 (2012), pp. 675-689. http://dx.doi.org/ $10.1007 / \mathrm{s} 00138-011-0362-8$.

[6] B. Galvin, B. McCane, K. Novins, D. Mason, and S. Mills, Recovering motion fields: An evaluation of eight optical flow algorithms, in Proceedings of the British Machine Vision Conference, BMVA Press, 1998, pp. 195-204. http://dx.doi.org/10.5244/C.12.20.

[7] W. Hackbusch, Iterative Solution of Large Sparse Systems of Equations, Springer, New York, 1993. ISBN 978-0-387-94064-9.

[8] B.K.P. Horn And B.G. Schunck, Determining optical flow, Artificial Intelligence, 17 (1981), pp. 185 - 203. http://dx.doi.org/10.1016/0004-3702(81)90024-2.

[9] J. Hubený, V. Ulman, And P. Matula, Estimating large local motion in live-cell imaging using variational optical flow, in Proceedings of the 2nd International Conference On Computer Vision Theory And Applications (VISAPP), 2007, pp. 542-548. ISBN 978-972-8865-74-0.

[10] B.D. LucAs AND T. KANADE, An iterative image registration technique with an application to stereo vision, in Proceedings of the 7th International Joint Conference on Artificial Intelligence (IJCAI), 1981, pp. 674-679.

[11] E. Meinhardt-Llopis, J. Sánchez PÉrez, and D. Kondermann, Horn-Schunck Optical Flow with a Multi-Scale Strategy, Image Processing On Line, 3 (2013), pp. 151-172. http: //dx.doi.org/10.5201/ipol.2013.20.

[12] J. Sánchez Pérez, N. Monzón López, and A. Salgado de la Nuez, Robust Optical Flow Estimation, Image Processing On Line, 3 (2013), pp. 252-270. http://dx.doi.org/10. 5201/ipol.2013.21.

[13] D. Sun, S. Roth, And M.J. Black, A quantitative analysis of current practices in optical flow estimation and the principles behind them, International Journal of Computer Vision, 106 (2014), pp. 115-137. http://dx.doi.org/10.1007/s11263-013-0644-x. 